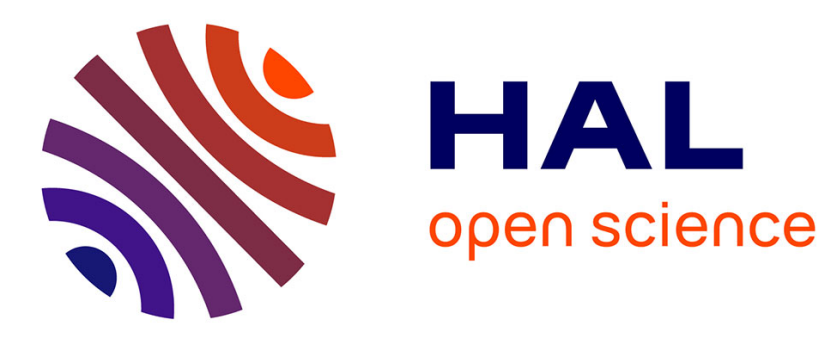

\title{
Hybrid Data Fusion Techniques for Localization in UWB Networks
}

Mohamed Laaraiedh, Stéphane Avrillon, Bernard Uguen

\section{To cite this version:}

Mohamed Laaraiedh, Stéphane Avrillon, Bernard Uguen. Hybrid Data Fusion Techniques for Localization in UWB Networks. WPNC'09, Mar 2009, Hannover, Germany. pp.51-57. hal-00375238

\section{HAL Id: hal-00375238 \\ https://hal.science/hal-00375238}

Submitted on 14 Apr 2009

HAL is a multi-disciplinary open access archive for the deposit and dissemination of scientific research documents, whether they are published or not. The documents may come from teaching and research institutions in France or abroad, or from public or private research centers.
L'archive ouverte pluridisciplinaire HAL, est destinée au dépôt et à la diffusion de documents scientifiques de niveau recherche, publiés ou non, émanant des établissements d'enseignement et de recherche français ou étrangers, des laboratoires publics ou privés. 


\title{
Hybrid Data Fusion Techniques for Localization in UWB Networks
}

\author{
M.Laaraiedh, S.Avrillon, B.Uguen \\ IETR, University of Rennes 1 \\ \{mohamed.laaraiedh, bernard.uguen, stephane.avrillon\}@univ-rennes1.fr
}

\begin{abstract}
In this paper, we exploit the concept of data fusion in UWB (Ultra Wide Band) localization systems by using different location-dependent observables. We combine ToA (Time of Arrival) and RSS (Received Signal Strength) in order to get accurate positioning algorithms. We assume that RSS observables are usually available and we study the effect of adding ToA observables on the positioning accuracy. The proposed architecture of Hybrid Data Fusion (HDF) is based on two stages: Ranging using RSS and ToA; and Estimation of position by the fusion of estimated ranges. In the first stage, we propose a new estimator of ranges from RSS observables assuming a path loss model. In the second stage, a new ML estimator is developed to merge different ranges with different variances. In order to evaluate these algorithms, simulations are carried out in a generic indoor environment and Cramer Rao Lower Bounds (CRLB) are investigated. Those algorithms show enhanced positioning results at reasonable noise levels.
\end{abstract}

Index Terms-Localization, RSS, ToA, Ranging, Weighted least square, Maximum Likelihood, Hybrid Data Fusion, UWB, Path Loss, Log Normal Shadowing, Cramer Rao Bound, GDOP.

\section{INTRODUCTION}

Nowadays, Location Based Services (LBSs) are more and more required by people and industries. Security is the main motivation for civilian mobile position location whose implementation is mandatory for the emergency calls. Besides security, the second leading application for wireless localization is intelligent transportation systems (ITSs). Personal tracking, navigation assistance and positiondependent billing are also new LBSs in expansion [1]. Furthermore, the location information is not only valuable for itself to provide new services but also to improve cellular communication systems at various levels. This is the scope of the FP7 WHERE project [2].

Positioning information can be, historically, obtained by trilateration and triangulation like in GPS [3] or roughly by getting the identity of the cell in a cellular networks. Many algorithms have been proposed to estimate the position of mobile station (MS) using simple location-depending observables (ToA, TDoA or RSS) as shown in [4], [5]. Nevertheless, these techniques cannot be used in the whole set of situations, especially when an observable is missing and cannot be readily obtained.

Thus, combining different location-dependent observables may achieve a better accuracy and simplify positioning system especially by reducing implementation complexity and/or communication overhead. In the case of indoor situations,
MS positioning can be based upon a short range radio access technology as UWB. The question hereafter addressed is how the MS or/and the network has to choose the appropriate combination of observables in order to achieve a required positioning accuracy.

In the context of UWB networks, ranging achieves good precision on ToA because of the used bandwidth which should be beyond $500 \mathrm{MHz}$ as defined by FCC [6]. In general, the observed ToA or TDoA error can be drastically reduced as the bandwidth increases beyond a certain value [7]. Moreover, RSS of each discovered node in the MS neighborhood is supposed to be always available. By default, position estimation based on RSS only can be continously performed but it can be very inaccurate. These properties motivate the choice of combining ToA with RSS to get enhanced positioning estimators.

In our assumed scenario, the MS is trying to continously obtain its position with the associated accuracy. The goal being the MS always adapts the measurements (type and number of added ToA) in order to perform positioning with the required accuracy. In order to minimize the consumption of ressources, the network seeks to find what type and how much of observables are accessible in a given context in order to achieve positioning. Thus, the chosen approach of this paper is to study the effect of adding ToA observables on the performances of positioning comparing with RSS only based positioning.

The rest of the paper is organized as follow. RSS-based ranging assuming a Log Normal Shadowing model and ToA-based ranging techniques are presented in section II. The algorithm based on the fusion of RSS with ToA is developed in section III. The formulation of the Cramer Rao Lower Bound (CRLB) is investigated in section IV. The performances of each algorithm are shown in section V. Finally, our concluding remarks are given in section VI.

In order to simplify the lecture of this paper, a list of abbreviations and symbols that are used in the paper is given in Table I.

\section{RSS-BASED RANGING VS TOA-BASED RANGING}

In this section, ToA-based and RSS-based ranging techniques are investigated. Assuming a Log Normal Shadowing model for path loss, a new ML estimator of ranges from RSS observables is proposed and described. 


\begin{tabular}{ll}
\hline AN & Anchor Node \\
LS & Least Square \\
ML & Maximum Likelihood \\
MS & Mobile Station \\
RSS & Received Signal Strength \\
$d$ & Distance between transmitter and receiver (m) \\
$d_{0}$ & Reference distance generally equal to 1 meter \\
$L$ & Pathloss at distance $d(\mathrm{~dB})$ \\
$L_{0}$ & Pathloss at distance $d_{0}(\mathrm{~dB})$ \\
$n_{p}$ & Pathloss exponent \\
$\lambda$ & Wavelength (m) \\
$\sigma_{s h}$ & Standard Deviation of shadowing (dB) \\
$\mathbf{x}=(x, y)$ & Coordinates of the MS \\
$\mathbf{x}_{k}=\left(x_{k}, y_{k}\right)$ & Coordinates of the $k^{t h}$ AN \\
$l$ & Length of the simulated area \\
$N_{\text {Trial }}$ & Number of Trials in Monte Carlo simulations \\
\hline
\end{tabular}

TABLE I: List of different used abbreviations and symbols.

\section{A. ToA-based ranging}

ToA-based ranging techniques are usually based on the estimate of the distance between transmitter and receiver from the ToA under the radio Line of Sight (LOS) assumption. To obtain this distance, many techniques are proposed such as One Way Ranging (OWR) shown in Fig. 1 and Two Way Ranging (TWR) shown in Fig. 2 where Tof is the Time of flight between the two sides of radio link and Dof is the associated Distance [8]. In [9], Power Delay Profile PDF) is also used in order to estimate ToA in Line of Sight (LoS) conditions. Nevertheless, ToA ranging is affected by relative clock drift between link sides, clock accuracy, the path loss model precision, and radio propagation phenomena such as shadowing, multipaths and non LoS conditions. [10].

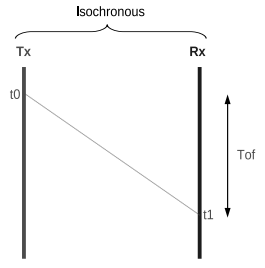

Fig. 1: ToA One Way Ranging

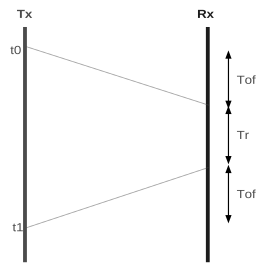

Fig. 2: ToA Two Way Ranging

\section{B. RSS-based ranging}

The simple analysis often used in coexistence studies limits the propagation characteristics to the large scale variation of the signal at given distances (pathloss). In mathematical terms, the mean received power $\bar{L}$ (around which there will still be shadowing and multipath) varies with distance following an exponential law. The total pathloss $\bar{L}$ at a distance $d$ is often modelled as [11]:

$$
\bar{L}=L_{0}+10 n_{p} \log \left(\frac{d}{d_{0}}\right)
$$

$d_{0}, d, n_{p}$ and $L_{0}$ are defined in Table I. $L_{0}$ is given by:

$$
L_{0}=20 \log \left(\frac{4 \pi d_{0}}{\lambda}\right)
$$

The measured loss varies about this mean according to a zero-mean Gaussian random variable, $X_{\sigma_{s h}}$, with standard deviation $\sigma_{s h}$. Shadowing is caused by obstacles between the transmitter and receiver that attenuate signal power through absorption, reflection, scattering, and diffraction. The complete path loss equation expressed in $\mathrm{dB}$ is then given by:

$$
L=L_{0}+10 n_{p} \log \left(\frac{d}{d_{0}}\right)+X_{\sigma_{s h}}
$$

This model can be used for both indoor and outdoor environments. For each environment or/and radio link, a characteristic value of each parameter, $n_{p}$ and $\sigma_{s h}$, is used. These values can be determined by calibration via measurement compaigns. Furthermore, the frequency and the bandwidth affect these parameters. The most common values of $n_{p}$ are shown in Table II for different types of environments.

\begin{tabular}{ll}
\hline Type of environment & Path loss exponent $n_{p}$ \\
\hline Free Space & 2 \\
Urban area cellular radio & 2.7 to 3.5 \\
Shadowed urban cellular radio & 3 to 5 \\
In building LOS & 1.6 to 1.8 \\
Obstructed in building & 4 to 6 \\
Obstructed in factory & 2 to 3 \\
\hline
\end{tabular}

TABLE II: Path Loss Exponent for different environments [6].

Let's consider the log normal shadowing described by the equation (3) as the used path loss model where we assume that the shadowing term $X_{\sigma_{s h}}$ is a zero-mean Gaussian r.v :

$$
X_{\sigma_{s h}} \sim \mathcal{N}\left(0, \sigma_{s h}^{2}\right)
$$

From (3) and (4) we derive the fact that the distance $d$ follows a Log-Normal distribution :

$$
p_{d}(d, L)=\frac{1}{\sqrt{2 \pi} d S} e^{\frac{-(\ln d-M)^{2}}{2 S^{2}}}
$$

where

$$
\begin{gathered}
S=\frac{\sigma_{s h} \ln 10}{10 n_{p}} \\
M=\frac{\left(L-L_{0}\right) \ln 10}{10 n_{p}}+\ln d_{0}
\end{gathered}
$$

As $d$ follows a Log-Normal distribution, the mean, median and mode estimators of distance $d$ are given respectively by [12]:

$$
\begin{aligned}
& \hat{d}_{L S}=e^{M+\frac{S^{2}}{2}} \\
& \hat{d}_{\text {median }}=e^{M}
\end{aligned}
$$




$$
\hat{d}_{M L}=e^{M-S^{2}}
$$

From equations (8) to (10), one can notice that the only estimator that does not require the knowledge of shadowing, given by the term $S$, is the median estimator. Thus, this estimator may be practical when no information about shadowing is available. Once the MS get this knowledge, the best estimator will be the mode which is the ML estimator. The mean estimator is not obviously a good choice as it overestimates the distance, and it is very inaccurate especially for strong values of $S$.

The knowledge of the associated variance of each estimator may enhance its performances. Theses variances must be included in each estimator of position in order to enhance the positioning accuracy. Thus, we derived for each estimator given by (8), (9) and (10) its variance. The expressions of these estimated variances are, respectively, given by:

$$
\begin{gathered}
\hat{\sigma}_{L S}^{2}=\hat{d}_{L S}^{2} e^{2 S^{2}}\left(e^{S^{2}}-1\right)=e^{2 M+3 S^{2}}\left(e^{S^{2}}-1\right) \\
\hat{\sigma}_{\text {median }}^{2}=\hat{d}_{\text {median }}^{2} e^{S^{2}}\left(e^{S^{2}}-1\right)=e^{2 M+S^{2}}\left(e^{S^{2}}-1\right) \\
\hat{\sigma}_{M L}^{2}=\hat{d}_{M L}^{2}\left(1-e^{-S^{2}}\right)=e^{2 M-2 S^{2}}\left(1-e^{-S^{2}}\right)
\end{gathered}
$$

One can remark that:

$$
\hat{\sigma}_{L S}^{2}>\hat{\sigma}_{\text {median }}^{2}>\hat{\sigma}_{M L}^{2}
$$

This property expresses the fact that the mode estimator is the best estimator of ranges from RSS observables. These variances can be advantageously exploited to improve the accuracy of RSS based positionning approach by providing a quantification of each estimated range reliability.

\section{Proposed New SCHEME OF Hybrid DATA Fusion OF RSS AND TOA}

The proposed system architecture is defined by a set of wireless nodes and one targeted MS to be positioned in a given indoor environment. The $\mathrm{K}$ wireless nodes $\left(A N_{k}\right)_{k=1 \ldots K}$ are placed in a squared room $\left(15 \times 15 \mathrm{~m}^{2}\right)$. These wireless nodes are assumed to be either anchor nodes or neighbor MSs able to compute their positions. In both cases, the node position is associated with a given level of precision. In this paper, this source of inaccuracy is neglected compared to the inaccuracy coming from observables. Thus, we suppose that all the nodes positions are well known with high precision.

The targeted MS for which the position estimation will be performed is assumed to be connected to the $K$ nodes. We consider that the link between MS and nodes is modeled according to the IEEE 802.15.4a standard with capability of ranging. All observables are coming from UWB Radio Access Network (RAN). Using this RAN, the MS is assumed to be able to get different types of observables (i.e RSS and ToA). As the RSS observables are usually accessible, we analyze the performances of $K-\mathrm{RSS} / m$-ToA based estimators using all the RSSs measured from the $K$ nodes and $m$ ToAs where $m$ varies in $(0, . ., K)$. We increase gradually the number of used ToAs in order to evaluate the contribution of these observables on positioning accuracy.

We consider the problem of estimating the unknown position $\mathbf{x}$ of the MS, exploiting the ranges obtained through RSS-based or ToA-based ranging procedures as described in section II with a set of $K$ anchor nodes $\mathbf{x}_{k}$. The output of ranging procedures is a set of ranges with their associated variances. We distinguish the following notations:

- true distance : $d_{k}=\left\|\mathbf{x}_{k}-\mathbf{x}\right\|$

- estimated range : $r_{k}$

- variance of estimated range $: \sigma_{r_{k}}^{2}$

The ranges $r_{k}$ are here supposed to be independent. If the true positions $\left[\mathbf{x}_{1}, \ldots, \mathbf{x}_{K}\right]$ of anchor nodes are perfectly known, we have the classical result that $\mathrm{ML}$ estimator is equivalent to the following minimization problem:

$$
\hat{\mathbf{x}}=\min _{\mathbf{x}} \sum_{k=1}^{K+m} \frac{\left(r_{k}-d_{k}(\mathbf{x})\right)^{2}}{\sigma_{r_{k}}^{2}}
$$

Let $\mathbf{F}$ be:

$$
\mathbf{F}=\sum_{k=1}^{K+m} \frac{\left(r_{k}-d_{k}(\mathbf{x})\right)^{2}}{\sigma_{r_{k}}^{2}}
$$

It can be readily shown that the ML estimator follows then the following implicit relation:

$$
\nabla \mathbf{F}=\sum_{k=1}^{K+m} \frac{1}{\sigma_{r_{k}}^{2}} \frac{\left(r_{k}-\left\|\mathbf{x}_{k}-\hat{\mathbf{x}}\right\|\right)}{\left\|\mathbf{x}_{k}-\hat{\mathbf{x}}\right\|}\left(\mathbf{x}_{k}-\hat{\mathbf{x}}\right)=\mathbf{0}
$$

Fig. 3 shows the proposed architecture of Hybrid Data Fusion (HDF) of RSS with ToA.

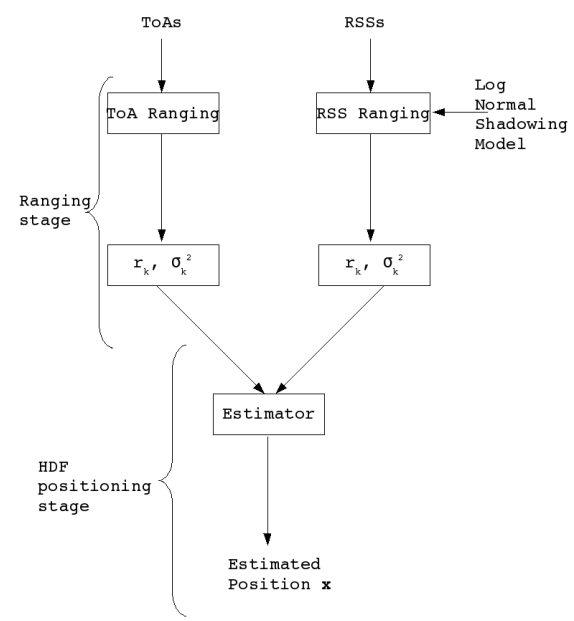

Fig. 3: Proposed architecure of HDF of RSS with ToA 


\section{CRamer Rao Bound of PRoposed NeW ESTIMATOR}

In this section, the mathematical formulation of the Cramer Rao Bound is presented. To proceed, let $\mathbf{J}(\hat{\mathbf{x}})$ defines the Fisher Information Matrix (FIM) for $\hat{\mathbf{x}}$. $\mathbf{J}(\hat{\mathbf{x}})$ is given by:

$$
\mathbf{J}(\hat{\mathbf{x}})=\mathrm{E}\left(\nabla \mathbf{F} \nabla \mathbf{F}^{T}\right)
$$

where $\nabla \mathbf{F}$ is given by the equation (17).

Let define the unit vectors between the estimated position $\hat{\mathbf{x}}$ and each known position of anchor nodes $\mathbf{x}_{k}$ as:

$$
\widehat{\mathbf{x x}}_{k}=\frac{\mathbf{x}_{k}-\hat{\mathbf{x}}}{\left\|\mathbf{x}_{k}-\hat{\mathbf{x}}\right\|}
$$

Thus, assuming an independence between the estimated ranges, $\mathbf{J}(\hat{\mathbf{x}})$ becomes:

$$
\begin{gathered}
\mathbf{J}(\hat{\mathbf{x}})=\mathrm{E}\left[\sum_{k=1}^{K+m} \frac{1}{\sigma_{r_{k}}^{4}}\left(r_{k}-\left\|\mathbf{x}_{k}-\hat{\mathbf{x}}\right\|\right)^{2} \widehat{\mathbf{x x}}_{k} \widehat{\mathbf{x x}}_{k}^{T}\right] \\
\mathbf{J}(\hat{\mathbf{x}})=\sum_{k=1}^{K+m} \frac{\widehat{\mathbf{x}}_{k} \widehat{\mathbf{x x}}_{k}^{T}}{\sigma_{r_{k}}^{2}}
\end{gathered}
$$

The CRLB of $\hat{\mathbf{x}}$ is defined by $\operatorname{cov}(\hat{\mathbf{x}}) \geq \mathbf{J}(\hat{\mathbf{x}})^{-1}$. Hence, the CRLB on the variance of the ToA/RSS location estimation is:

$$
\sigma_{C R L B}^{2}=\min (\operatorname{tr}(\operatorname{cov}(\hat{\mathbf{x}})))=\operatorname{tr}\left(\mathbf{J}(\hat{\mathbf{x}})^{-1}\right)
$$

Developping (22) leads to this expression of $\sigma_{C R L B}^{2}$ :

$$
\sigma_{C R L B}^{2}=\frac{\sum_{i=1}^{K+m} \frac{1}{\sigma_{r_{i}}^{2}}}{\sum_{i=1}^{K+m} \sum_{j \neq i}^{K}\left(\frac{\left(x-x_{i}\right)^{2}\left(y-y_{j}\right)^{2}}{\sigma_{r_{i}}^{2} \sigma_{r_{j}}^{2}\left\|\mathbf{x}_{i}-\hat{\mathbf{x}}\right\|^{2}\left\|\mathbf{x}_{j}-\hat{\mathbf{x}}\right\|^{2}}\right)}
$$

\section{Simulation Results and Discussions}

In this section, we evaluate the performances of the proposed HDF scheme described in section II and III through Monte Carlo simulations. Two criteria are chosen to perform the evaluation: Positioning error and CRLB.

\section{A. Positioning error}

The different steps of the simulation are the following:

1) $K$ random ANs and one targeted MS are uniformly drawn in an area of $l \times l \mathrm{~m}^{2}$.

2) Different path losses $\left(L-L_{0}\right)$ are computed for each link $k$ between the MS and the $k^{t h}$ AN. For each link, log normal shadowing model is applied with randomly drawn values of $n_{p}, \lambda$ and $\sigma_{s h}$. Table III shows the parameters used in simulations.

3) A Gaussian random range is drawn centred on the true distance between the MS and respectively $m$ ANs where $m$ varies in $(0, . ., K)$. The values of the variance of ToA, $\sigma_{T}^{2}$, are also drawn randomly and are given in Table III. $m$ is increased gradually to show the effect of ToAs on positioning accuracy.

\begin{tabular}{ll}
\hline Parameters & Values in Indoor \\
\hline$n_{p}$ & 1.6 to 1.8 \\
$\lambda(m)$ & 0.12 \\
$\sigma_{s h}(d B)$ & 2 to 5 \\
$\sigma_{T}(n s)$ & 0 to 6.67 \\
$l(m)$ & 15 \\
\hline
\end{tabular}

TABLE III: List of radio parameters used in simulations for indoor scenario.

4) All simulations have been done with a number of trials equal to $N_{\text {Trial }}=1000$.

$n_{p}, \sigma_{s h}$ and $\sigma_{T}$ are choosen randomly different from one link to other in order to better simulate the behaviour of radio propagation channel. In fact, in a real radio channel these parameters depend on many factors like visibility between the transmitter and the receiver, number and type of materials, used frequencies and many other factors [6].

In Fig. 4, the cumulative density functions of three different estimators are plotted. In this figure, we assume that only RSS observables are available (i.e $m=0$ ). Ranges are then obtained by either mean, median or mode estimators described in section II. The proposed estimator (17) is then applied on these ranges in order to estimate position. This figure shows that the performances of the new proposed estimator depend on the used estimator of ranges from RSSs. The mode estimator performs better than median and mean estimators. In the rest of this section, performances are evaluated assuming the mode estimator of ranges from RSS observables. In Fig. 5, the new proposed estimator (17) is compared to typical weighted LS estimator based on trilateration and the linearization of at least three circle equations in 2D space [13]. The figure shows how much the proposed estimator performs better than the weighted LS estimator. Comparing to the typical weighted LS position estimator, the proposed new estimator merges smartly the different observables with different associated variances, and overcomes positioning error caused by the stage of linearization [14].

In Fig. 6, the effect of additional ToAs on the positioning accuracy is shown. In UWB networks, ToA ranging is generally very accurate. Thus, adding more accurate ToAs enhances the positioning accuracy. The figure confirms this remark. Indeed, the cumulative density is as better as the number of additional ToAs is greater. Nevertheless, the figure shows that adding ToAs can deteriorate the performances comparing to RSS based positioning. The reasons of this deterioration may be either the bad precision of measured ToA or the high Geometric Dilution of Precision (GDoP) [15]. In fact, the GDoP is frequently thought of as a number signifying the effect of anchor nodes geometry on computed position [16]. These observations motivate the necessity of defining a criterion for choosing the best placed nodes with which the MS should carry out a ToA based ranging. We believe that this criterion will depend on the configuration of the nodes and the precision of ranges. We suggest the Cramer Rao Lower Bound as a good criterion to choose the adequate number, 
type and position of measurements for a given scenario. In the next subsection, its properties which motivate our choice are described based on the results of simulations.

\section{B. Cramer Rao Lower Bound}

The Cramer Rao Lower Bound (CRLB) sets a lower bound of any unbiased estimator. This can be very useful in localization algorithms. The CRLB can provide a benchmark against which we can compare the performance of any unbiased estimator [15].

Simulations are done respecting these rules:

1) Four anchor nodes are first placed in the corners of an $(l \times l)$ area.

2) For each point in the $(l \times l)$ area, we compute the CRLB for different scenarios (4 RSSs $+m$ ToAs).

3) Used radio parameters are given in Table IV.

\begin{tabular}{ll}
\hline Parameters & Used values \\
\hline$n_{p}$ & 1.7 \\
$\lambda(m)$ & 0.12 \\
$\sigma_{s h}(d B)$ & 2 \\
$\sigma_{T}(n s)$ & 3.33 \\
$l(m)$ & 15 \\
\hline
\end{tabular}

TABLE IV: List of radio parameters used in CRLB simulations for indoor scenario.

The figures from Fig. 7 to Fig. 12 plot the CRLB over the $(l \times l)$ area. These figures confirm the results shown in Fig. 6 that additional ToAs enhance the positioning accuracy. In particular, comparison between Fig. 7 and Fig. 12 reveals that the fusion of RSS and ToA mitigates the difficulties of locating devices in the proximity of the anchor nodes. This result is also obtained by Catovic in [17] even when considering relative positioning (i.e assuming the interdistances between anchor nodes are known).

Moreover, these figures show that the choice of the number of additional ToAs may affect the positioning accuracy. In Fig. 8 and Fig. 9, the same number of additional ToAs $(m=1)$ is used but with different positions of associated anchor nodes. The comparison between these two figures reveals that the values of CRLB, in each point of the area, are different even when the same number of additional ToAs is used. Thus, the configuration of involved anchor nodes in the ToA based ranging can enhance or deteriorate the accuracy on a given MS location. This can be very useful in the case of on-demand location based services where the user seeks the service with the satisfying accuracy. The number and associated position of additional ToAs may define the requested level of accuracy.

Fig. 13 shows the average CRLB for different schemes of RSS/ToA positioning over the entire area for varying sizes of the area. This figure reveals that, despite the given global enhancement, additional ToAs reduce the effect of the area size on the positioning accuracy. Indeed, the average CRLB of $4 \mathrm{RSS} / 3 \mathrm{ToA}$ and $4 \mathrm{RSS} / 4 \mathrm{To} \mathrm{A}$ are almost quasi-independent

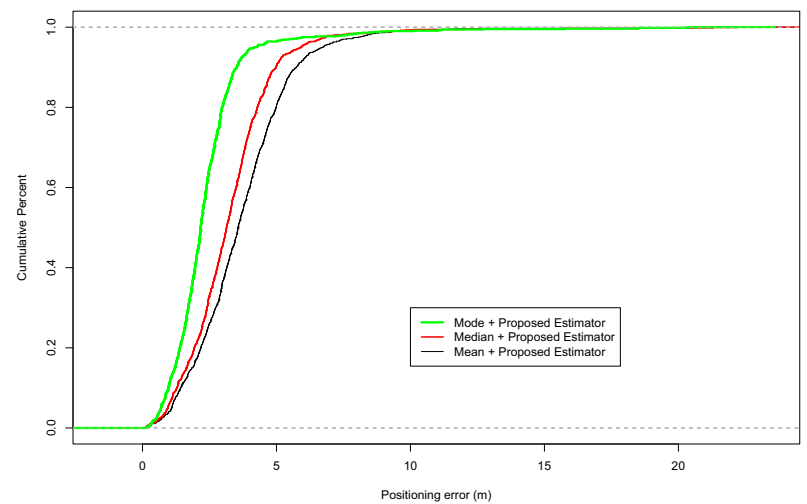

Fig. 4: Compared cumulative density function of different studied RSS based ranging estimators for indoor scenario $-l=15 \mathrm{~m}, K=$ 4 .

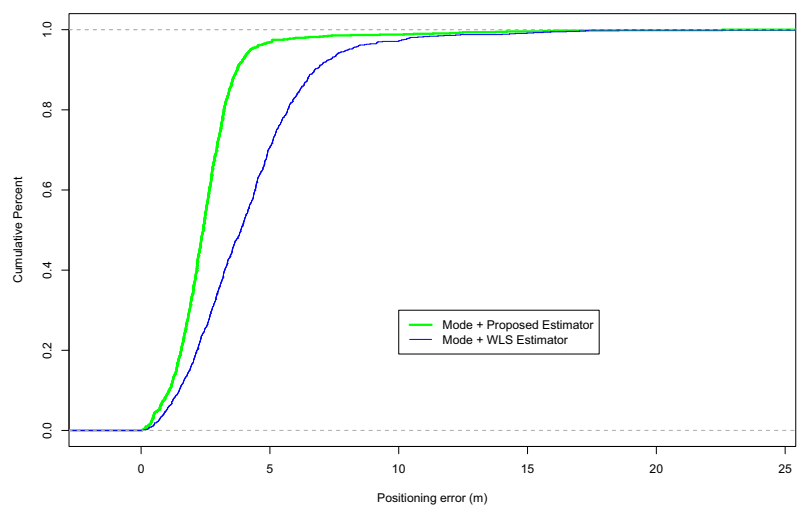

Fig. 5: Comparison between cumulative density functions of new proposed estimator and weighted least square for indoor scenario - $l=15 m, K=4$.

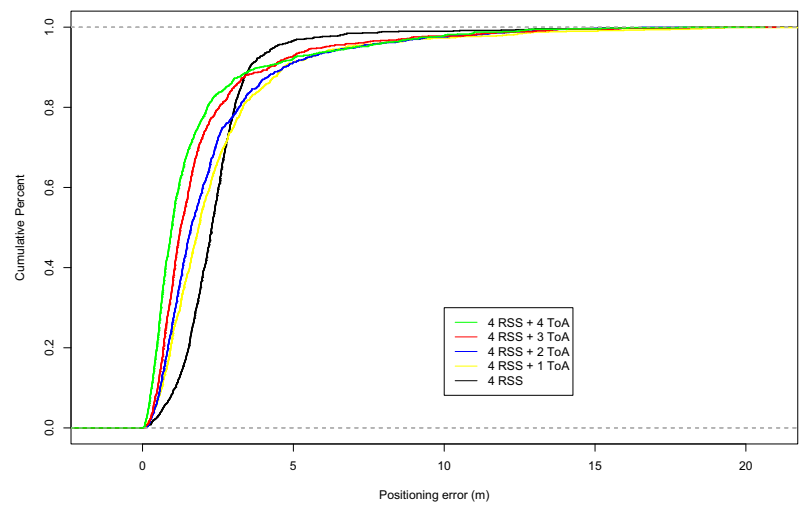

Fig. 6: Effect of additional ToA on cumulative density function of the proposed estimator for indoor scenario $-l=15 \mathrm{~m}, K=4$. 
of the size of the area.

In conclusion, the Cramer Rao Lower Bound presents a good criterion to choose the best number, type, and associated positions of observables in order to perform a service with the required accuracy. We suggest that this criterion must be involved in tracking system giving, hence, the capability of choosing the best configurations of anchor nodes and observables. Thus, the system may enhance the performed services, reduce the cost of implementation, and preserve the ressources.

\section{CONCLUSION}

In this paper, we proposed an hybrid RSS/ToA localization scheme based on two steps: First step consists in estimating ranges from RSS assuming a path loss log-normal shadowing model and from ToA based on two way and one way ranging techniques. Second step consists in estimating the position using these previously estimated ranges. In the first step, we proposed a new estimator of ranges and associated variances from RSS observables. We believe that this technique enhances the accuracy of RSS-based ranging and consequently the positioning accuracy. In the second step, we proposed a new ML estimator able to merge smartly different ranges while considering associated variances. This estimator performs better than typical weighted least square estimator. The effect of additional ToAs on the performances is studied using positioning error and Cramer Rao Lower Bound. We believe that the number and the position of anchor nodes implied in ToA-based ranges must be smartly choosen in order to perform the requested positioning accuracy. Next step will be to evaluate performance in more realistic scenarios and especially by using more realistic path loss model with adequate parameters.

\section{ACKNOWLEDGMENT}

The work presented in this paper has been performed in the framework of the ICT-217033 WHERE project funded by the European Union.

\section{REFERENCES}

[1] P. Bellavista, A. Kupper, and S. Helal, "Location-based services: Back to the future," IEEE, Pervasive Computing, 2008.

[2] "http://www.kn-s.dlr.de/where/."

[3] Y. Chan and K. Cho, "A simple and efficient estimator for hyperbolic location," IEEE Transactions On Signal Processing, vol. 42, Aug. 1994.

[4] G. Shen, R. Zetik, and R. Thoma, "Performance comparison of toa and tdoa based location estimation algorithms in los environment," WPNC 08, 2008.

[5] K. Cheung, H. So, W. Ma, and Y. Chan, "A constrained least squares approach to mobile positioning: Algorithms and optimality," EURASIP journal on applied signal processing, no. 17, 2006.

[6] A. Goldsmith, Wireless Communications. cambridge university press ed., 2005.

[7] S. Gezici, Z. Tian, G. B. Biannakis, H. Kobayashi, A. F. Molisch, V. Poor, and Z. Sahinoglu, "Localization via ultra-wideband radios," IEEE Signal Processing Magazine, vol. 22, July 2005.

[8] C. Wen, J. Chen, and W. Sethares, "Asynchronous two-way ranging using tomlinson-harashima precoding and uwb signaling," EURASIP Journal on Wireless Communications and Networking, 2008.

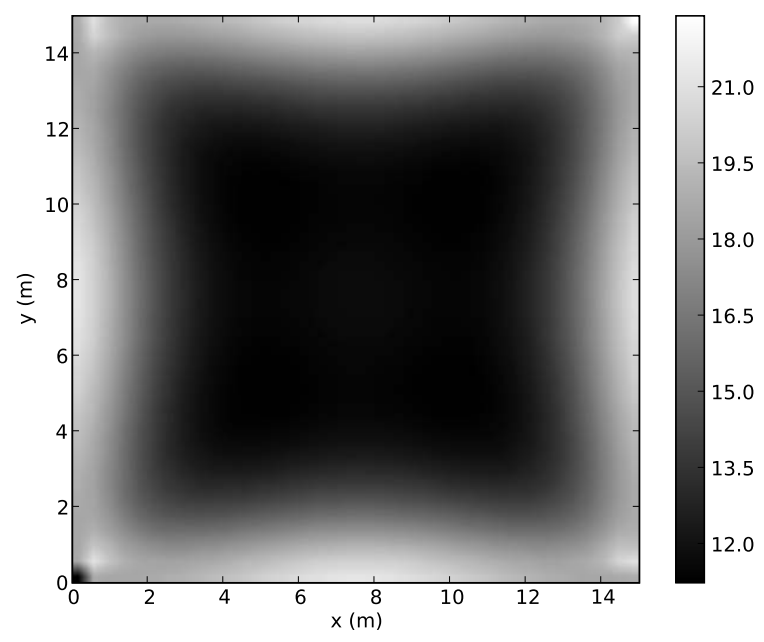

Fig. 7: Cramer Rao Lower Bound for 4 RSSs - $l=15 m, K=4$.

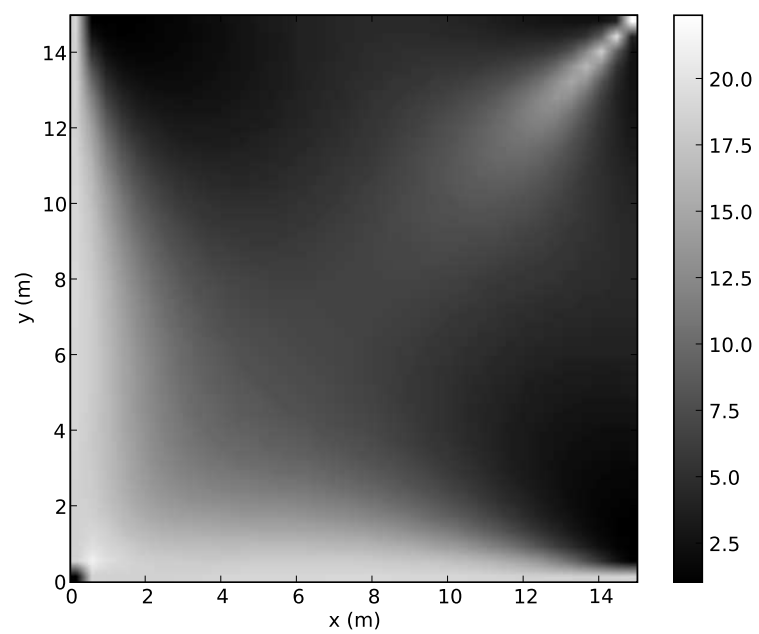

Fig. 8: Cramer Rao Lower Bound for 4 RSSs/ 1 ToAs [0.0,0.0]$l=15 m, K=4$.

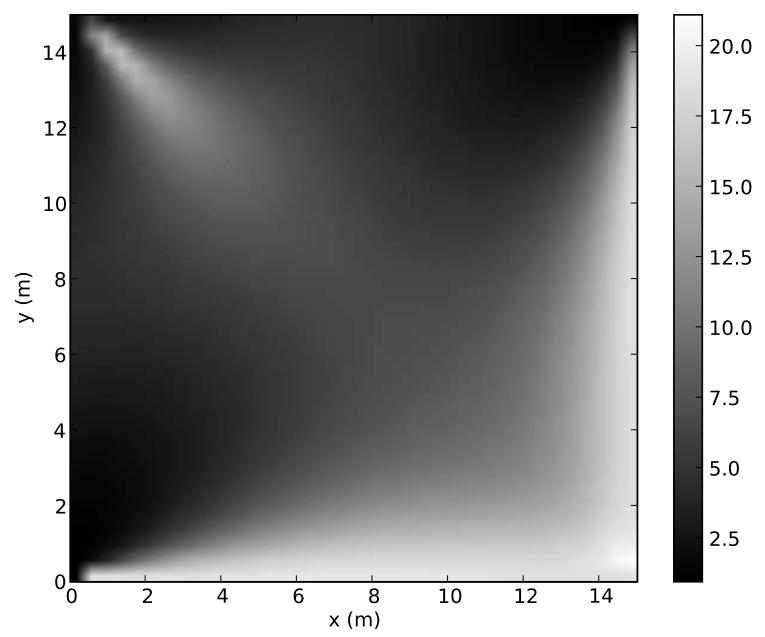

Fig. 9: Cramer Rao Lower Bound for 4 RSSs/ 1 ToAs [15.0,0.0] $l=15 m, K=4$. 


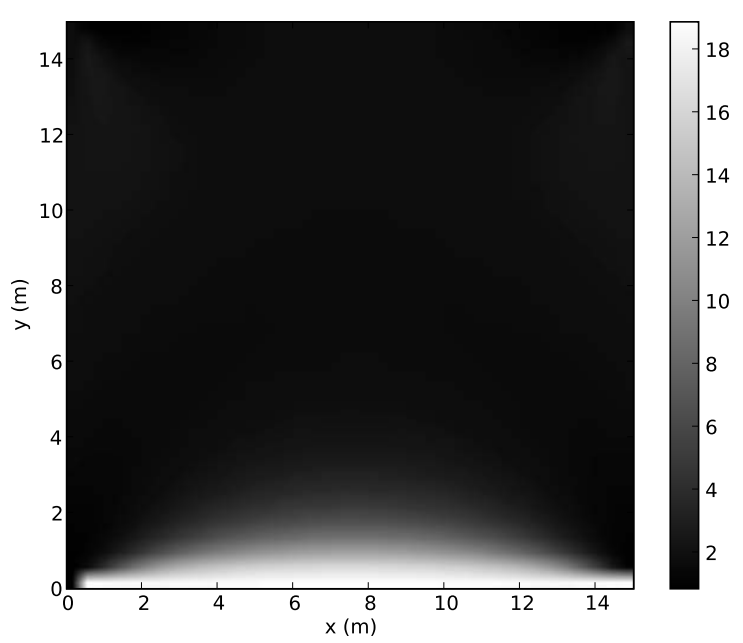

Fig. 10: Cramer Rao Lower Bound for 4 RSSs/ 2 ToAs $-l=$ $15 m, K=4$.

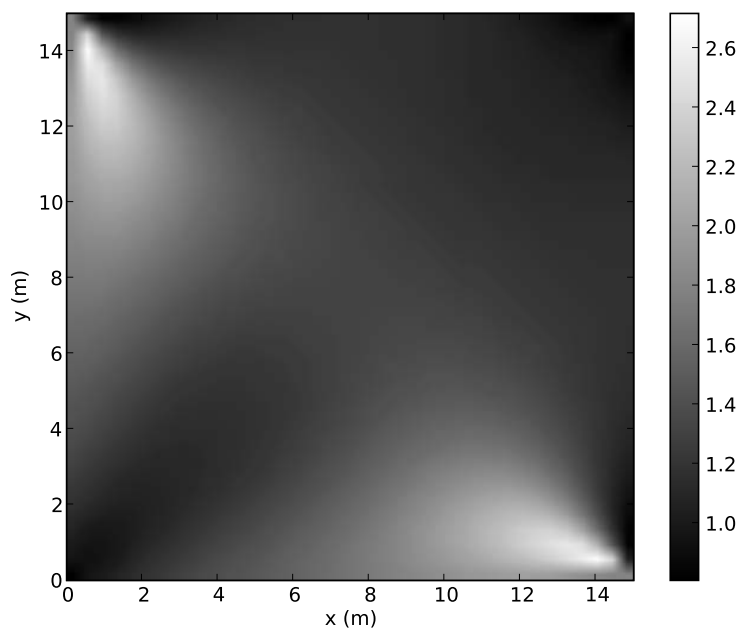

Fig. 11: Cramer Rao Lower Bound for 4 RSSs/ 3 ToAs $-l=$ $15 m, K=4$.

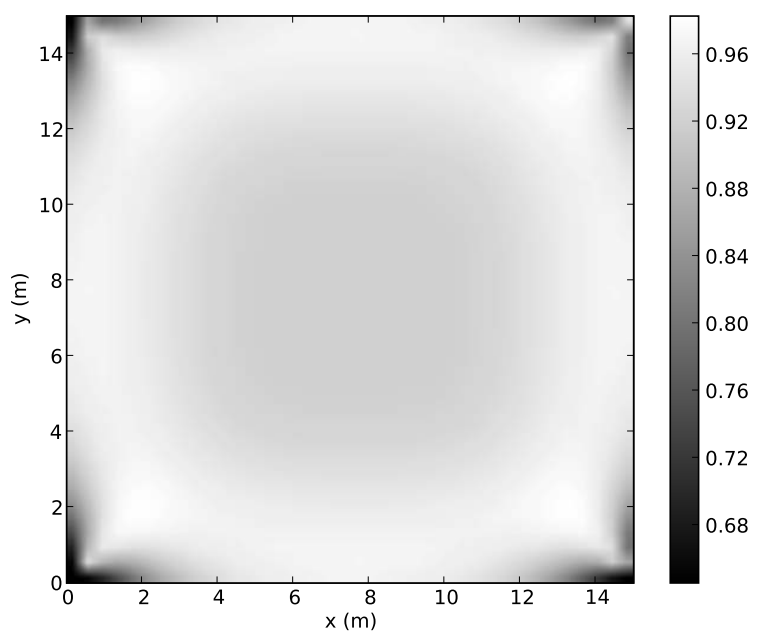

Fig. 12: Cramer Rao Lower Bound for 4 RSSs/ 4 ToAs - $l=$ $15 m, K=4$.

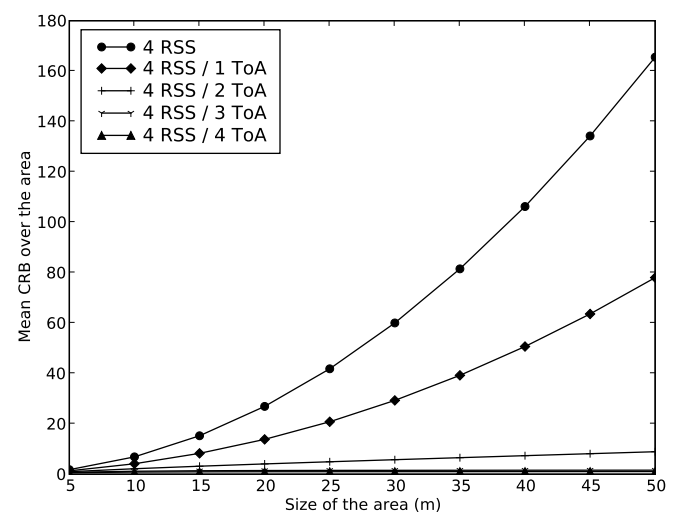

Fig. 13: Variation of the Cramer Rao Lower Bounds with respect to the size of the area for different scenarios - $K=4$.

[9] C. Mazzucco, U. Spagnolini, and G. Mulas, "A ranging technique for uwb indoor channel based on power delay profile analysis," IEEE VTC Spring, 2004.

[10] B. Denis, J. Keignart, and N. Daniele, "Impact of nlos propagation upon ranging precision in uwb systems," IEEE Conference on Ultra Wideband Systems and Technologies, 2003.

[11] V. Abhayawardhana, W. Crosby, M. Sellars, and M. Brown, "Comparison of empirical propagation path loss models for fixed wireless access systems," VTC 2005 spring, vol. 1, 2005.

[12] M. P.McLaughlin, A Compendium of Common Probability Distributions, vol. Regress+ Documentation. 1999.

[13] M. Laaraiedh, S. Avrillon, and B. Uguen, "Enhancing positioning accuracy through direct position estimators based on hybrid rss data fusion," IEEE VTC Spring, 2009.

[14] W. Kim, J. Lee, and G. Jee, "The interior-point method for an optimal treatment of bias in trilateration location," IEEE Transactions on Vehicular Technology, vol. 55, July 2006.

[15] N. Patwari, A. I. Hero, M. Perkins, N. Correal, and R. O'Dea, "Relative location estimation in wireless sensor networks," IEEE Transactions On Signal Processing, vol. 51, pp. 2137-2148, Aug. 2003.

[16] J. Chaffee and J. Abel, "Gdop and the cramer-rao bound," IEEE Position Location and Navigation Symposium, Apr. 1994.

[17] A. Catovic and Z. Sahinoglu, "The cramer-rao bounds of hybrid toa/rss and tdoa/rss location estimation schemes," Communications Letters, IEEE, vol. 8, no. 10, pp. 626-628, Oct. 2004. 